\title{
Diffractive photoproduction of lepton pairs at high energy
}

\section{Gabriela Ślipek*}

Institute of Nuclear Physics PAN, PL-31-342 Cracow, Poland,

E-mail: Gabriela.Slipek@ifj.edu.pl

\section{W. Schäfer ${ }^{1}$, A. Szczurek ${ }^{1,2}$}

${ }^{1}$ Institute of Nuclear Physics PAN, PL-31-342 Cracow,Poland,

${ }^{2}$ University of Rzeszów, PL-35-959 Rzeszów,Poland

E-mail: Wolfgang.Schafereifj.edu.pl,Antoni.Szczurek@ifj.edu.p

We derive the forward photoproduction amplitude for the diffractive $\gamma p \rightarrow l^{+} l^{-} p$ reaction in the momentum space within the formalism of $k_{\perp}$ - factorization. Using this formalism we study exclusive photoproduction of lepton pairs $\gamma p \rightarrow l^{+} l^{-} p$ at high energies $W_{\gamma p}>100 \mathrm{GeV}$.

We calculate the total cross section as a function of photon-proton center of mass energy and the invariant mass distribution of the lepton pair. We also discuss whether the production of timelike virtual photons can be approximated by continuing to the spacelike domain $q^{2}<0$. The amplitude will be used to predict the cross section for exclusive production of lepton pairs in the $p p \rightarrow p l^{+} l^{-} p$ reaction.

XVIII International Workshop on Deep-Inelastic Scattering and Related Subjects, DIS 2010 April 19-23, 2010

Firenze, Italy

\footnotetext{
* Speaker.
} 


\section{Introduction}

Measuring absolutely normalized cross sections at the LHC is of great importance for the high-energy physics community. This requires having a well understood luminosity monitor. The QED process $p p \rightarrow p l^{+} l^{-} p$ via photon-photon fusion is often discussed as a process which can be used for measuring the luminosity at the LHC. It is therefore very important to estimate other nonQED contributions to exclusive $l^{+} l^{-}$production. One possible source of dileptons is the exclusive production of vector mesons or $Z$-bosons (see e.g. [1, 2, 3]).

In the present work we shall concentrate on the photon-pomeron subprocess. In Fig.1 we show a QCD mechanism, where the photon splits into a quark-antiquark pair which interacts with the proton through the exchange of an off-diagonal QCD gluon ladder. In Fig.1 the incoming photon is spacelike, (or quasireal) but the outgoing photon is timelike, i.e. $q^{2}>0$. Here we review the momentum-space formulation of timelike Compton scattering at small- $x$ introduced in [4] which takes into account the timelike nature of the final state photon.

\section{Formalism}

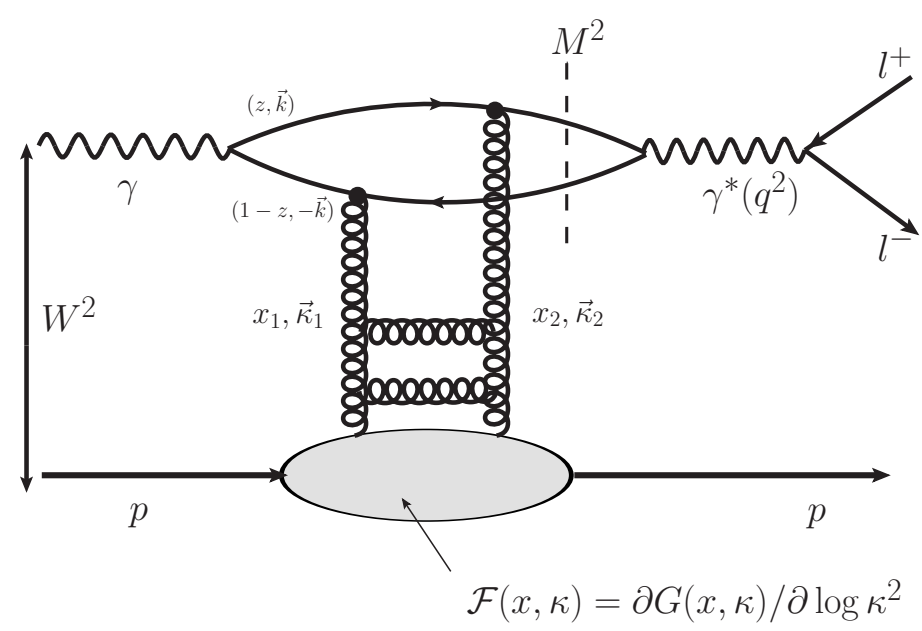

Figure 1: The diagram for the production of virtual timelike photons.

The photoproduction amplitude will be the major building block for our prediction of exclusive dilepton pair production in $p p$ collisions. The amplitude for the $\gamma p \rightarrow l^{+} l^{-} p$ reaction is shown schematically in Fig.1. The calculation of the amplitude follows the same procedure as for the exclusive production of vector mesons, which is explained in detail in Ivanov's thesis [5]. The main difference is that the final state light-cone wavefunction is replaced by a free quark propagator times the QED-spinor structure for the $q \bar{q} \rightarrow \gamma^{*}$ transition. The amplitude of the subprocess $\gamma p \rightarrow$ $\gamma^{*}\left(q^{2}\right) p$ is a sum of the contributions for a given flavour $f$ of quarks in the loop. The forward $\gamma p \rightarrow \gamma^{*} p$ amplitude for a given flavour contribution can then be written as:

$$
\mathscr{M}_{f}\left(\gamma p \rightarrow \gamma^{*}\left(q^{2}\right) p\right)=W^{2} 4 \pi \alpha_{\mathrm{em}} e_{f}^{2} 2 \cdot 2 \int_{0}^{1 / 2} \frac{d z}{z(1-z)} \int_{0}^{\infty} \pi d k_{\perp}^{2} \frac{\mathscr{A}_{f}\left(z, k_{\perp}^{2}, W^{2}\right)}{\left[\frac{k_{\perp}^{2}+m_{f}^{2}}{z(1-z)}-q^{2}-i \varepsilon\right]},
$$


where the $\mathscr{A}_{f}\left(z, k_{\perp}^{2}, W^{2}\right)$ we refer to it as the glue convolution, $\alpha_{\mathrm{em}}$ is the QED fine-structure constant; $e_{f}=\frac{2}{3}$ for $u, c, t$ and $e_{f}=-\frac{1}{3}$ for $d, s, b$ is the quark charge. The quark masses $\left(m_{f}\right)$ have been fixed for: $m_{u, d}=0.22 \mathrm{GeV}, m_{s}=0.37 \mathrm{GeV}, m_{c}=1.5 \mathrm{GeV}, m_{b}=4.75 \mathrm{GeV}$. The masses used here are consistent with the analysis of deep inelastic scattering data in Ref.[6]. This analysis was used to find the unintegrated gluon distribution which is used in the present analysis. The transverse momentum squared of (anti-)quarks is denoted by $k_{\perp}^{2}$, their longitudinal momentum fractions are $z$ and $1-z$, respectively. The running coupling $\alpha_{s}$ enters at the scale $q^{2}=\max \left\{\kappa^{2}, k_{\perp}^{2}+m_{f}^{2}\right\}$, where $m_{f}$ is the quark mass for flavor $f$. Now notice, that the invariant mass of the $q \bar{q}$ pair is given by

$$
M^{2}=\frac{k_{\perp}^{2}+m_{f}^{2}}{z(1-z)}
$$

so that the second line of Eq.(2.1) suggests a change of variables from $\left(z, k_{\perp}^{2}\right) \rightarrow\left(M^{2}, k_{\perp}^{2}\right)$. Finally, we can cast the amplitude in the form

$$
\mathscr{M}_{f}\left(\gamma p \rightarrow \gamma^{*}\left(q^{2}\right) p\right)=W^{2} 16 \pi^{2} \alpha_{\mathrm{em}} e_{f}^{2} \cdot \int_{4 m_{f}^{2}}^{\infty} \frac{a_{f}\left(W^{2}, M^{2}\right)}{M^{2}-q^{2}-i \varepsilon} d M^{2}
$$

Here $a_{f}\left(W^{2}, M^{2}\right)$ is related to the diffractive amplitude for the $\gamma p \rightarrow q \bar{q} p$ transition [7], however with the spinorial contractions from the final state performed. We refer to it as the spectral function. Very detailed discussion concerning the spectral function can be found in our original paper [4].

As a last step we must extend our amplitude to finite momentum transfers. For simplicity we will assume it to have the following factorized form

$$
\mathscr{M}_{f}\left(\gamma p \rightarrow \gamma^{*}\left(q^{2}\right) p ; t\right)=\mathscr{M}_{f}\left(\gamma p \rightarrow \gamma^{*}\left(q^{2}\right) p\right) \exp [B t] .
$$

The total cross section for the $\gamma p \rightarrow \gamma^{*} p$ process is obtained as

$$
\sigma\left(\gamma p \rightarrow \gamma^{*}\left(q^{2}\right) p\right)=\frac{\left(\Re e \frac{\mathscr{M}}{W^{2}}\right)^{2}+\left(\mathfrak{I} m \frac{\mathscr{M}}{W^{2}}\right)^{2}}{16 \pi B},
$$

where for definiteness we take $B=4 \mathrm{GeV}^{-2}$.

The invariant mass distribution of dileptons for the $\gamma p \rightarrow l^{+} l^{-} p$ reaction is given by

$$
\frac{d \sigma}{d q^{2}}\left(\gamma p \rightarrow l^{+} l^{-} p\right)=\frac{\alpha_{\mathrm{em}}}{3 \pi q^{2}} \cdot \sigma\left(\gamma p \rightarrow \gamma^{*}\left(q^{2}\right) p\right)
$$

where $l^{+} l^{-}$means either $e^{+} e^{-}$or $\mu^{+} \mu^{-}$. This simple formula applies only when $q^{2} \gg m_{l}^{2}$. More detailed discussion of the formalism can be found in [4].

\section{Results}

Here we present predictions for the $\gamma p \rightarrow l^{+} l^{-} p$ reaction. In Fig. 2 we show the invariant mass distribution $d \sigma / d q^{2}$ of dileptons in the $\gamma p \rightarrow l^{+} l^{-} p$ reaction as a function of photon-proton center-of-mass energy at fixed values of the invariant masses of the dilepton pairs $\left(q^{2}\right)$. Here all flavours are included in the amplitude. In general, the higher invariant mass, the faster growth with 


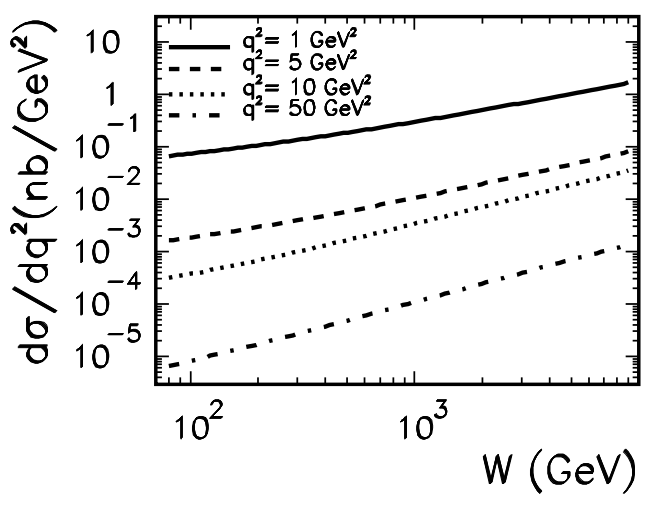

Figure 2: The cross section for $\gamma p \rightarrow l^{+} l^{-} p$ as a function of center-of-mass photon-proton energy for fixed values of the dilepton invariant mass.

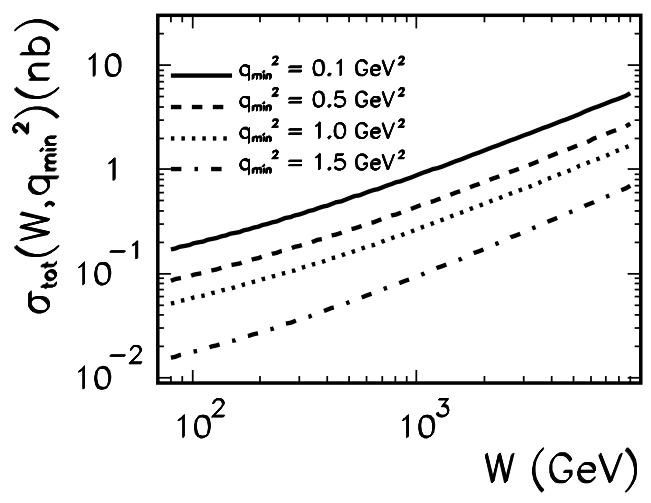

Figure 3: $\sigma_{t o t}\left(\gamma p \rightarrow l^{+} l^{-} p ; q_{\min }^{2}\right)$ for $q_{\min }^{2}=0.1,0.5,1.0,1.5 \mathrm{GeV}^{2}$.

the photon-proton energy. This points to the fact that the unintegrated glue is probed at on average harder scales, where it has a faster $x$-dependence. In Fig. 3 we show cross section integrated over dilepton invariant mass

$$
\sigma_{t o t}\left(\gamma p \rightarrow l^{+} l^{-} p ; q_{\min }^{2}\right)=\int_{q_{\min }^{2}}^{\infty} \frac{d \sigma}{d q^{2}} d q^{2}
$$

These cross sections are by a factor of about 5 larger than those in [8].

Finally, it is interesting to investigate how well the dilepton mass spectrum can be calculated from the amplitude for the production of spacelike photons in the final state. In Fig. 4 we show the ratio of $d \sigma / d q^{2}$ for the timelike photons and the spacelike prescription (used previously in the literature) as a function of $q^{2}$ at various fixed energies. We observe that, the spacelike prescription does not reproduce the structure present in the timelike amplitude. The cross section for timelike 


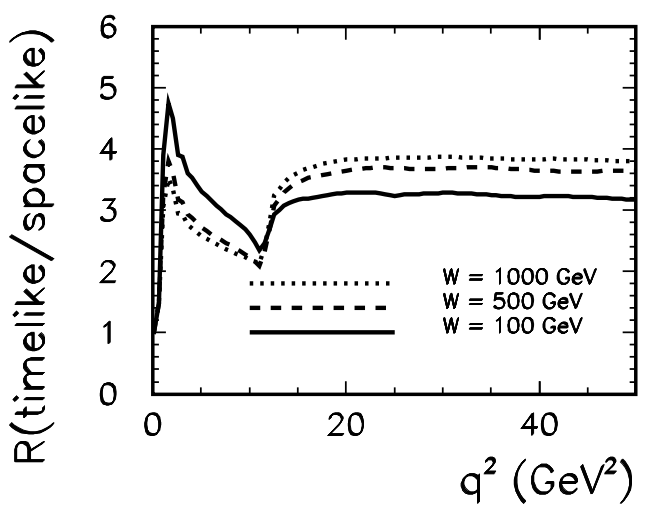

Figure 4: The ratio of the cross section $\frac{\frac{d \sigma}{d q^{2}}(\text { timelike })}{\frac{d \sigma}{d q^{2}}(\text { spacelike })}$ as a function of $q^{2}$.

photons is bigger by a factor of 3-4 compared to the spacelike photon prescription.

\section{Conclusions}

We have derived the amplitude for the exclusive diffractive photoproduction of lepton pairs in the $k_{\perp}$-factorisation approach in the momentum space. We have discussed several details of the formalism as well as differences compared to the existing calculation in the literature which ignored the fact that the "produced" photons are timelike.

We have calculated the cross sections as a function of photon-proton center of mass energy as well as a function of dilepton invariant mass. We have demonstrated how important is the inclusion of correct dynamics (timelike outgoing photons instead of spacelike outgoing photons).

The amplitude for the $\gamma p \rightarrow l^{+} l^{-} p$ is the main ingredient of the diffractive amplitude for the $p p \rightarrow l^{+} l^{-} p p$ process.

Acknowledments This work was supported by the MNiSW grants: N N202 236937.

\section{References}

[1] W. Schäfer and A. Szczurek, Phys. Rev. D76 (2007) 094014.

[2] A. Rybarska, W. Schäfer and A. Szczurek, Phys. Lett. B668,126 (2008).

[3] A. Cisek, W. Schäfer and A. Szczurek, Phys. Rev. D80 (2009) 074013.

[4] W. Schäfer, G. Ślipek and A. Szczurek, Phys. Lett. B688,185 (2010)

[5] I.P. Ivanov, [arXiv:hep-ph/0303053].

[6] I.P. Ivanov and N.N. Nikolaev, Phys. Rev. D65 (2002) 054004.

[7] N. Nikolaev and B.G. Zakharov, Z. Phys. C 53 (1992) 331; Phys. Lett. B 332 (1994) 177.

[8] M.V.T. Machado, Phys. Rev. D78 (2008) 034016. 